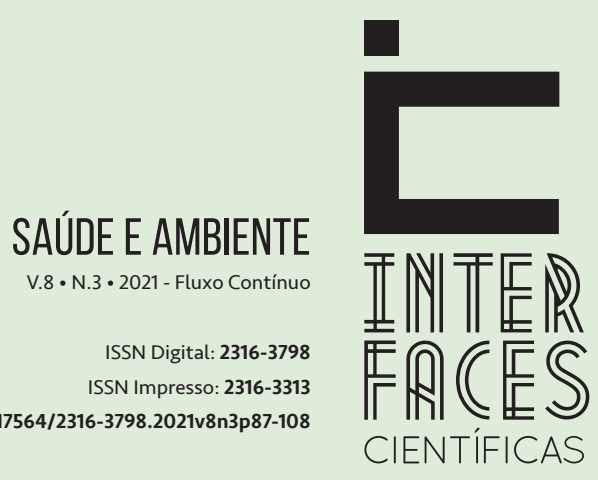

\section{ANÁLISIS SOCIO CRÍTICO DE LOS COMEDORES COMUNITARIOS EN EL ESTADO DE MÉXICO: LA SUMISIÓN SOCIAL ANTE EL HAMBRE}

ANÁLISE SOCIAL CRITIICA DOS REFEITÓRIOS COMUNITÁRIOS NO ESTADO DO MÉXICO SUBMISSÃO SOCIAL NA FACE DA FOME

SOCIO-CRITICAL ANALYSIS OF COMMUNITY CANTEENS IN THE STATE OF MEXICO: SOCIAL SUBMISSION TO HUNGER

Donovan Casas Patiño ${ }^{1}$ Fernando Castro Grifaldo ${ }^{2}$ Alejandra Rodríguez Torres ${ }^{3}$ Georgina Contreras Landgrave

\section{RESUMEN}

El programa de Comedores Comunitarios (CC), fue una política contra el hambre, teniendo como objetivo el mejorar las condiciones de acceso a la alimentación de la población, existe evidencia de intereses político-económicos, pasando a segundo plano los propósitos para lo cual habían sido instalados. Es un estudio de corte cualitativo, creando y aplicando una entrevista de tipo semiestructurada en la Zona Oriente del Estado de México. Este estudio tuvo como objetivo analizar desde una postura socio crítica al programa CC de la Zona Oriente del Estado de México, el análisis discursivo fue con la ayuda del software Iramuteq V7. El CC está asociado al factor económico, buenas perspectivas y opiniones, los colectivos refuerzan la necesidad de los CC como parte fundamental de su desarrollo. Los CC son una ayuda alimentaria paliativa del hambre, el dinamismo del núcleo discursivo parte de la economía como un apoyo social y proteccionismo estatal.

\section{PALABRAS CLAVE}

Comedores Comunitarios, Hambre, Teoría socio crítica, Dieta. 


\section{RESUMO}

O programa Refeitórios Comunitários (RC) era uma política contra a fome, com o objetivo de melhorar as condições de acesso à alimentação da população, evidenciando-se interesses político-econômicos, ficando em segundo plano os propósitos para tal que foi instalado. É um estudo qualitativo, elaborando e aplicando uma entrevista semiestruturada na Zona Leste do Estado do México. Este estudo teve por objetivo analisar o programa RC da Zona Leste do Estado do México a partir de uma posição sociocrítica. A análise discursiva se deu com o auxílio do software Iramuteq V7. O RC está associado ao fator econômico, boas perspectivas e opiniões, os grupos reforçam a necessidade do CC como parte fundamental do seu desenvolvimento. Os RC são um auxílio alimentar paliativo para a fome, o dinamismo do núcleo discursivo faz parte da economia como suporte social e protecionismo estatal.

\section{PALAVRAS-CHAVE}

Refeitórios Comunitários. Fome. Teoria Sociocrítica. Dieta.

\section{ABSTRACT}

The Community Dining Hall (CC) program was a policy against hunger, with the objective of improving the conditions of access to food for the population, there is evidence of political-economic interests, passing the purposes for the background which had been installed. It is a qualitative study, creating and applying a semi-structured interview in the Eastern Zone of the State of Mexico. This study aimed to analyze from a socio-critical position the CC program of the Eastern Zone of the State of Mexico, the discursive analysis was with the help of the Iramuteq V7 software. The CC is associated with the economic factor, good perspectives and opinions, the groups reinforce the need of the CC as a fundamental part of their development. The $\mathrm{CC}$ are a palliative food aid for hunger, the dynamism of the discursive core part of the economy as a social support and state protectionism.

\section{KEYWORDS}

Community Dining Hall. Hunger. Socio-critical Theory. Diet.

Interfaces Científicas - Aracaju • V.8 • N.3 • p. 87 - 108 • 2021 - Fluxo Contínuo 


\section{INTRODUCCIÓN}

El programa social denominado "Comedores Comunitarios" (CC) inició actividades en el año 2013. Este programa asistencial alimentario es uno de los más grandes de la época del siglo XXI en México, su origen fue justificado por las cifras alarmantes de pobreza; señaladas según el informe del Consejo Nacional de Evaluación de la Política de Desarrollo Social (Coneval), donde, el porcentaje de personas en condición de pobreza alimentaria (insuciencia para adquirir una canasta básica de alimentos) pasó de 13.8\% (14.4 millones) en el 2006 a 18.2\% (19.5 millones) en el 2008, (CONEVAL, 2009). Para el 2012, México tenía 53.3 millones de personas en condición de pobreza (45.5\%), incrementando la carencia por acceso a la alimentación a 27.4 millones, poco más de una quinta parte de la población nacional (CONEVAL, 2012).

Por lo anterior, en el Plan Nacional de Desarrollo 2013-2018 emitido por la administración presidencial de Enrique Peña Nieto, estableció la primera política social masiva denominada Cruzada Nacional Contra el Hambre (SINHAMBRE). Esta constaba de cinco puntos principales: a) Cero hambre a partir de una alimentación y nutrición adecuada de personas en pobreza extrema y con carencia por acceso a la alimentación, b) Eliminar la desnutrición infantil aguda, c) Aumentar la producción de alimentos y el ingreso de campesinos y pequeños productores agrícolas, d) Minimizar pérdidas de alimentos desde la postcosecha hasta la comercialización. e) Promover la participación comunitaria para la erradicación del hambre". (SECRETARÍA, 2013, p. 14).

Los encargados de ejecutar esta política fueron la Secretaria de Desarrollo Social (SEDESOL), el Consejo Nacional, el Comité de Alimentación (académicos expertos en alimentación), los Comités Comunitarios y los Acuerdos Integrales para el Desarrollo Social (entre órdenes de gobierno). Sumado a los puntos anteriormente descritos, se debería de garantizar el derecho a la educación, la salud, la alimentación nutritiva y de calidad, la vivienda, el disfrute de un medio ambiente sano, el trabajo y la seguridad social, así como la no discriminación; todo esto para más de $50 \%$ de la población mexicana (DÍAZ, 2018).

Es así, que el hambre se convirtió oficialmente según el Estado en un enemigo de lucha al mismo tiempo que permitiría mostrar el compromiso social y la unidad en contra del hambre como prioridad nacional. México fundamento un elemento de combate a este mal mediante la SEDESOL con un presupuesto de 251, 168, 286 pesos mexicanos (12, 589, 559.17 USD) en el 2013, y el 2018 con 3, 205, 757,494 pesos mexicanos (160, 685, 388.63 USD) (MARTíNEZ, 2019).

Así para el año 2017 existían en todo México 5,294 CC pertenecientes a SEDESOL (Figura 1), de estos, una gran parte se concentraba en el Estado de México (2000 CC) que ofrecían en un día común 311 mil raciones diarias (CONEVAL, 2017), siendo la entidad federativa con más CC. Esto no fue coincidencia, se trata de un estado con una población necesitada y marginada, creando coerción mediática de un Estado preocupado por la necesidad del que menos tiene, procurando tener un número muy alto de CC, para control y posición de estas poblaciones marginadas (BOURDIEU, 2014). 
Figura 1 - Organograma de estructural: Programa Comedores Comunitarios

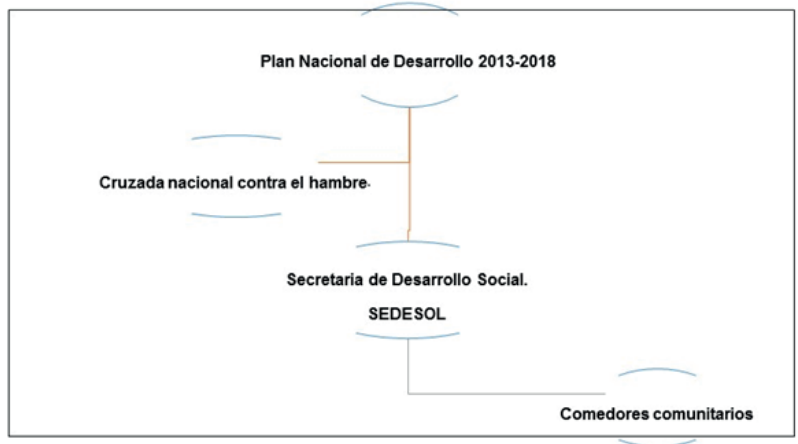

Fuente: Propria.

El objetivo general de los CC fue mejorar las condiciones de acceso a la alimentación de la población ubicada en Zonas de Atención Prioritaria (ZAP) rurales o urbanas (Cuadro 1), a través del acceso a la alimentación (HERNÁNDEZ et al., 2015). Dentro de su población objetivo se encontraban: niñas/os de 0 a 11 años de edad, estudiantes adolescentes de 12 a 19 años, mujeres embarazadas y en periodo de lactancia, población que sufriera contingencias o emergencias que requirieran una intervención excepcional, personas con alguna discapacidad, adultas mayores de 65 años, en situación de vulnerabilidad y pobreza.

Cuadro 1 - Zonas de Atención Prioritaria (ZAP)

\begin{tabular}{|c|c|}
\hline ZAP Rural & ZAP Urbana \\
\hline $\begin{array}{c}\text { 1,080 municipios que se encuentran en 26 } \\
\text { entidades federativas, y que cumplen con } \\
\text { alguna de las siguientes condiciones: son } \\
\text { de Muy Alta o Alta Marginación, tienen un } \\
\begin{array}{c}\text { Muy Alto o Alto Índice de Rezago Social o al } \\
\text { menos el 25\% de la población se encuentra } \\
\text { en pobreza multidimensional extrema. }\end{array}\end{array}$ & $\begin{array}{c}\text { 8,139 Áreas Geoestadísticas Básicas (AGEBs) urbanas } \\
\text { incluye a un total de 6.54 millones de hogares censa- } \\
\text { les y que cumplen las siguientes condiciones: AGEBs } \\
\text { urbanas con Muy Alto o Alto Grado de Marginación e } \\
\text { Indice de Rezago Social Bajo, Medio y Alto, adicional- } \\
\text { mente las AGEBs urbanas con Índice de Rezago Social } \\
\text { Alto o Medio y Grado de Marginación Medio. }\end{array}$ \\
\hline
\end{tabular}

Fuente: propia, con información de DOF (2017).

El equipamiento de los CC (Figura 2), fue realizado por SEDESOL y DICONSA 5* $^{*}$ teniendo un costo hasta de 150 mil pesos (7,518.68 USD) (DOF, 2014). Cada CC se abastecía mensualmente de produc-

$5^{*}$ Diconsa (DIstribuidora CONasupo, SA) opera el Programa de Abasto Rural con más de 27 mil tiendas fijas y 300 móviles en todo el país, para cumplir con su objetivo cuenta con 302 almacenes rurales y centrales, 3 almacenes graneleros y casi 4 mil vehículos que cada día recorren miles de kilómetros de carreteras y terracerías. Tiene como objetivo garantizar el abasto oportuno de productos básicos y complementarios, a precios accesibles, en localidades rurales de alta y muy alta marginación, participando con transparencia y eficacia en su desarrollo de acuerdo a su cultura y entorno ecológico, bajo un esquema de corresponsabilidad entre proveedores, trabajadores, instancia de participación social y gobierno. 
tos no perecederos por medio de DICONSA (frijol, aceite, lentejas, barra de frutas, arroz, soya, huevo en polvo, jugo de fruta, carne deshidratada de res, sopas, etc.), teniendo un cálculo para 120 beneficiarios, con un valor máximo mensual de 65,000 pesos (3,258.06 USD) por cada CC. Esta cifra al dividirse entre los 20 días de servicio del CC mensual, entre120 beneficiarios nos da un total de 27.0 pesos al día (1.35 USD) para cada usuario, teniendo como salario mínimo en esa fecha un promedio de $\$ 80$ pesos, esto para dos tiempos de alimentación (desayuno y comida).

Figura 2 - Equipamientos de los Comedores Comunitarios

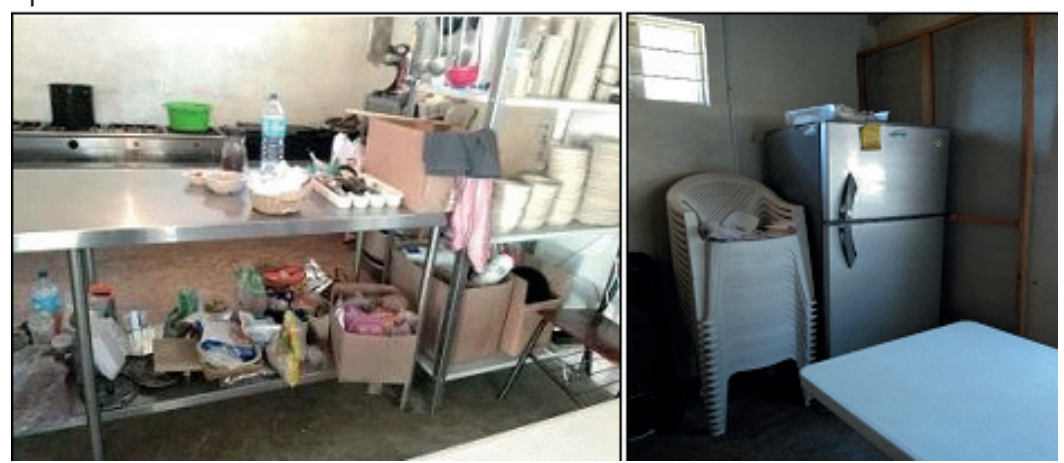

Fuente: Propia. Comedor Comunitario Atlautla, Estado de México.

Su función era ofrecer dos tiempos de alimentación (desayuno de 9 a 10 am y comida de 2 a 3 pm) en los cuales existía una cuota de recuperación de 10 pesos por ración. La Secretaría de Marina (SEMAR) o Secretaría de la Defensa Nacional (SEDENA) se encargaron de la capacitación inicial a los integrantes del comité comunitario, esto en aspectos básicos como el manejo y preparación de las raciones alimentarias (HERNÁNDEZ et al., 2015). La dieta regularmente consistía en café o té, frijoles en sus diferentes presentaciones (fritos, molidos, de la olla), pasta, arroz, carne seca (res), fruta enlatada o deshidratada, entre otros (Figura 3). 
Figura 3 - Función. Ejemplos de desayuno y comida
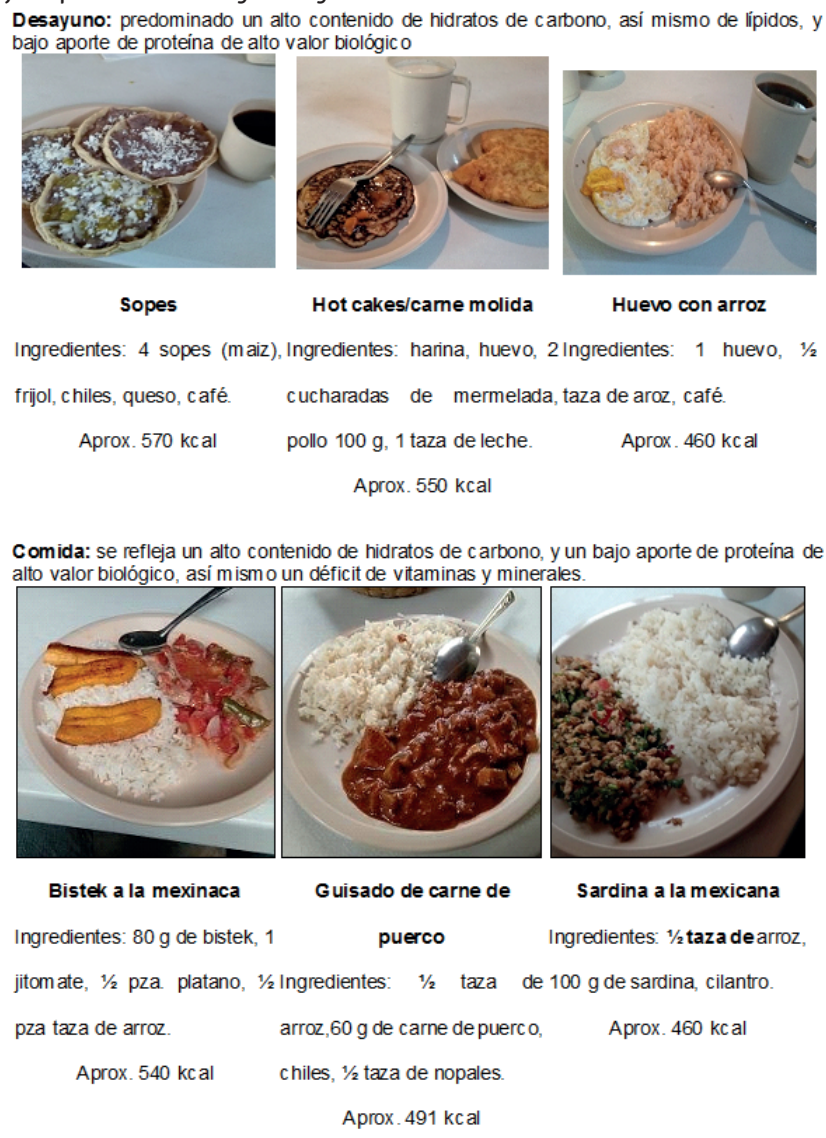

Fuente: Propia. Comedor Comunitario Amecameca / Atlautla. Kcal obtenidas a través del sistema mexicano de alimentos equivalentes.

La estructura organizacional dentro del CC estaba constituida en un comité comunitario integrado por la comunidad y representados por una mesa directiva: Presidente, un Secretario, un Tesorero, Vocales de Comisiones de Trabajo y un Vocal de Control y Vigilancia, preferentemente de forma equitativa entre hombres y mujeres, todos ellos elegidos democráticamente en Asamblea General Constitutiva (SECRETARIA DE BIENESTAR, 2019).

Este tipo de políticas sociales de asistencialismo han sido construidas para intereses muy particulares del Estado, los programas públicos a menudo pasan por alto los beneficios populares (ZAMUDIO et al., 2018), al respecto, los CC y la cruzada Nacional Contra el Hambre permitieron al Estado posicionarse ventajosamente en la escena internacional para contribuir con los objetivos del milenio y por otro lado "ofrecieron la posibilidad de utilizarlo para fines electorales, policiales y judiciales"(LUTZ; 
TORRES, 2016 p. 171), además las industrias alimentarias y de la sociedad civil difundieron un buen modelo del buen comer, incorporando alimentos procesados en la dieta de los beneficiarios trastocando bases culinarias ancestrales (TORRES; LUTZ, 2016). También existen tendencias económicas neoliberales que favorecen patrones de alimentación cada vez más homogéneos y que amenazan la Seguridad Alimentaria Nacional (GARZA et al., 2018), y además se demostró que las condiciones higiénicas e infraestructura no son adecuadas en los CC, las dietas no cubrían las calorías para cada grupo de edad, y los menús contenían alimentos saludables pero la cantidad no era ideal (BALAM et al., 2013), por lo cual los CC en México perpetuaron conductas populistas de asistencialismo popular para beneficio político (CANSINO; COVARRUBIAS, 2007).

Los CC han tenido presencia a nivel internacional, esto por crisis hiperinflacionarias como en la Argentina (LERULLO, 2013), además en ellos existe una creciente cultura normativa de dietas insalubres, considerando esto un factor que contribuye a la mala nutrición, hay también evidencia de relaciones entre la obesidad y la ingesta nutricional deficiente en estos $\mathrm{CC}$, además no existe evidencia científica respecto a la percepción y aceptación de estos programas en poblaciones de riesgo de inseguridad alimentaria (LOOPSTRA; TARASUK, 2013; MILLS et al., 2017; HEBINCK et al., 2018).

Cabe mencionar, que el Estado jugó un papel importante y decisivo para la planeación y ejecución de las políticas sociales de beneficencia de los CC, creando las condiciones necesarias que garantizaran el derecho de una alimentación saludable, acceso diario, de calidad, digno y en cantidad, para atender necesidades nutricionales básicas de los colectivos, el acto alimenticio reconstruye y dignifica hábitos sociales determinados por un colectivo. Es así que los CC se pueden entender como una política social masiva que busca beneficios propios para el Estado - clientelismo electoral, populismo, militancia partidista, entre otros-, objetivando al Hambre como el enemigo social. Por lo cual el objetivo de esta investigación fue analizar desde una postura socio crítica al programa CC, siendo fundamental conocer la realidad social.

\section{METODOLOGÍA}

\subsection{MARCO TEÓRICO}

Se utiliza un anclaje teórico para esta investigación, en este caso paradigma socio crítico, con el fin de obtener un análisis socio crítico que devele la realidad social en torno a los comedores comunitarios desde una perspectiva del usuario.

La teoría crítica se originó de la Escuela de Frankfurt en la década de 1920 bajo la dirección autoritaria del filósofo Max Horkheimer, Theodor Adorno, Otto Kirchheimer, Erich Fromm, Walter Benjamin (primera generación). Y posteriormente en los años 70 fue retomada por, entre otros (segunda generación), y que para fines de esta investigación se comprende la teoría critica de Jürgen Habermas desde un enfoque sociopolítico.

Partiendo de un análisis descriptivo y comprensivo de las estructuras sociales, gestión y procesos mediante una reflexión y concientización, logrando con ello una emancipación de la sociedad, 
haciendo una crítica al orden social establecido y perpetuado. Siendo un elemento focal del análisis socio crítico la Emancipación, definida como: "autonomía o capacidad del individuo de reflexionar sobre la realidad para responder así a las injusticias y desigualdades de la sociedad"(HABERMAS, 1982 apud FERNÁNDEZ, 1995, p. 246). Para ello un punto importante es conocer la realidad social del programa de comedores comunitarios, sus relaciones implícitas y no implícitas, donde la incertidumbre y el conflicto, que son partes únicas de la construcción en el tiempo de los colectivos, y que son capaces de mostrar o develar la vida mismas de las personas y su relación con los entramados sociales.

Los sujetos o colectivos están constituidos socialmente en una RED de tramas de signos, símbolos, significaciones y perpetuaciones de poder y régimen, por ello pueden ser analizados e interpretados en un contexto de anclaje socio-histórico. Y las formas que se dan en los niveles macro (desde la lucha contra el hambre a nivel internacional) meso (la cruzada nacional contra el hambre) y micro (el programa comedores comunitarios frente a los colectivos), anclados en los elementos como: política, economía, cultura, educación y sociedad. Lo que permitirá llegar a una panóptica de observación la cual nos ofrece una visión a la realidad social encontrando que el programa es distante a la finalidad de origen.

Desde la praxis fue fundamental y de impetuosa necesidad anclar los discursos de los usuarios para generar un análisis apegado a la realidad de su creación, servicio, utilidad, etc., en un referente de perspectiva usuaria a partir de identidad, accesibilidad, calidad de la dieta, políticas del servicio, cultura alimentaria e intercambio de redes socioculturales.

\subsection{MARCO METODOLÓGICO}

Es un estudio cualitativo, trasversal y descriptivo, esta investigación se realizó en los CC de 3 municipios de la Zona Oriente del Estado de México Atlautla, Amecameca, Tepetlixpa; la selección de los participantes estuvo guiada por criterios de conveniencia, oportunidad y disponibilidad (OSSA; MUÑOZ, 2015); así mismo por los criterios de inclusión: personas que hayan firmado el consentimiento informado, personas que acuden al comedor comunitario con regularidad, personas mayores de 18 años de edad. El tamaño de la muestra fue no probabilístico y estuvo determinado mediante la saturación discursiva (MARTÍNEZ, 2012; SERBIA, 2019).

Se realizó en un acercamiento micro-etnográfico de acuerdo a Jiménez y colaboradores (2017) a los comedores comunitarios, con la finalidad de conocer y contextualizar los espacios y discursos en torno al comedor comunitario, y recolectar información para una entrevista semiestructurada a partir de un grupo focal de 5 usuarios obteniéndose discursos y se procedió a construir categorías analíticas, las cuales dieron pauta para la construcción de la entrevista semiestructurada (CASTRONUOVO et al., 2016). La entrevista semiestructurada fue validada mediante la técnica de juicio de expertos (del área de investigación cualitativa) en ronda de tres, incluyo inicialmente 105 ítems, los pasos metodológicos quedaron de la siguiente forma:

1. se obtuvo un instrumento final de 51 ítems, que incluyen las categorías: identidad, accesibilidad, calidad de la dieta, políticas del servicio, cultura alimentaria e intercambio de redes socioculturales, este instrumento se aplicó a 23 usuarios de CC;

2. después que se trascribieron las entrevistas, se enumeraron las unidades de importancia (en 
general, sujeto y predicado);

3. se organizan las palabras según el orden de aparición en el discurso, posteriormente de identifican cueles con las que más se repiten;

4. se realizó la frecuencia y porcentajes de presentación, y una asociación de palabras discursivas con el software Iramuteq V7.

5. se sometió al Comité Local Multidisciplinario de Investigacion Nezahualcóyotl, con número de registro 2019-38-BN.

\section{RESULTADOS Y ANÁLISIS}

Los resultados obtenidos de 23 usuarios; 16 fueron del sexo femenino y 7 sexo masculino, predomina el sexo femenino, existe una sumisión de este grupo, al rol social de cuidado del hogar, de niños y ancianos, este rol es asumido por influencia social (MOCTEZUMA et al., 2014; ROSAS 2019), donde además, está asignado el rol de preparación de comida, siendo invisibilizados sus derechos desde perspectiva de género (VELÁZQUEZ et al., 2014). Acorde a ocupación; 9 amas de casa, 3 estudiantes, 1 instructora de gimnasio, 8 empleo informal, 2 profesoras (primaria y secundaria), predomina el ser ama de casa y el empleo informal, la causas el rol social asignado por género y "el bajo ritmo de crecimiento que ha presentado el país" (MARTíNEZ, 2018).

La edad promedio fue de 51.43 años, en México de los 123.5 millones de personas, 65.2 millones tienen menos de 29 años (NOTIMEX, 2017), respecto a esto, es poca la presencia de niños y jóvenes en los CC. En cuanto al estado civil; 14 casadas, 3 divorciadas, 4 solteras, y 2 viudas, aunque el matrimonio ya no es una sociedad económica y la preocupación por la felicidad de manera individual es adoptada como rol de vida (CASAS; RODRÍGUEZ, 2018), en estas comunidades prexiste la idea del matrimonio como estatus social. En relación al nivel educativo: 5 licenciatura, 5 preparatoria, 7 secundaria y 6 primaria; esto refleja un bajo nivel educativo, en relación a ello, existen causas para no continuar con los estudios, predominando las de tipo económico, personales, y sociales (GÓMEZ, 2017).

El ingreso económico promedio mensual fue de 6,117 pesos (313.19 USD), concuerda con el salario promedio mensual en México (JIMÉNEZ, 2018), pero cabe mencionar que el precio de la canasta básica es de 10,925.17 pesos (47.58 USD) (MORA, 2019), lo cual no puede ser adquirida en su totalidad.

En relación a salud, 8 personas presentan sobrepeso u obesidad, 5 hipertensión arterial, 4 diabetes mellitus y 3 sanas, se observa la presencia de Enfermedades Crónico no Transmisibles (ECNT) en los usuarios de CC, esto concuerda con datos oficiales: 10 de 7 personas adultas cursan con sobrepeso u obesidad, 1 de cada 4 cursa con hipertensión arterial y 7 de 10 cursa con diabetes mellitus (ENSANUT, 2016), se observa que el CC debería ser una propuesta de combate a ECNT.

Finalmente religión; 1 cristiana, 2 mormonas (Santos de los Últimos Días), 18 católicos, 1 libre pensador, 1 practicante de la religión Maya; lo anterior muestra un predominio del catolicismo, siendo la más profesada por los mexicanos (APARICIO, 2015) acorde a lo anterior, la relevancia de conocer la religión reside en la adaptación del enfoque de programas sociales sobre cierta población, ya que la 
religión parte de un sistema de doctrinas, practicas e instituciones, por medio de códigos morales y símbolos de comunicación (INEGI, 2009), permitiendo la integración social, a partir de un proceso histórico que ha desempeñado un papel condicionante sobre las elecciones alimentarias a través de juicios de valor (CONTRERAS, 2007; DÍAZ, 2015), es así que la religión aglutina en torno a este programa social.

Para la interpretación y asociación discursiva de los usuarios en relación a los CC, se realizó un análisis discursivo de las categorías: identidad, accesibilidad, calidad de la dieta, políticas del servicio, cultura alimentaria e intercambio de redes socioculturales (Cuadro 2). Posteriormente se esquematizaron los análisis a través de Iramuteq V7, para focalizar núcleo y periferia de las relaciones discursivas en torno a la realidad social del programa CC por medio de las categorías analíticas, obteniendo un corpus (Figura 4).

Cuadro 2 - Discursos respecto a las categorías de análisis de usuarios del programa CC

\begin{tabular}{|c|c|}
\hline Identidad & Accesibilidad \\
\hline $\begin{array}{l}\text { E1, E2, E4,E6, E7, E8, E9, E11, E13, E15, } \\
\text { E16, E18, E19, E20, E23 “personas de } \\
\text { bajos recursoses un apoyo a la economía } \\
\text { el no gastar tanto”, E3, E11, E12, E13, } \\
\text { E18 “brindan alimento”, E4, E5,E6, E15, } \\
\text { E16, E17 E19, E21, E3, E5, E8, E11, E13, } \\
\text { E18, E22 “todo tipo de personasniños, } \\
\text { adultos”, E7, E8, E10, E13, E15, E17, E19, } \\
\text { E22, E23 “para apoyar a la poblaciónun } \\
\text { apoyo a la población en general a bajo } \\
\text { costo” E12, E14, E20 “alimentación es un } \\
\text { poco más completapara la nutrición”, E1, } \\
\text { E10, E11, E19, E22 “ahorrar tiempo”. }\end{array}$ & $\begin{array}{l}\text { E1, E5, E6, E7, E8, E9, E10, E14, E16, E18, E19 “está bien } \\
\text { ahorita está bien” E6, E12, E13, E19, es libre porque es co- } \\
\text { munitario”, E3, E8, E9, E12, E18, E19 “personas que tienen } \\
\text { bajos recursos económicos...las personas pobres es una } \\
\text { población con más necesidad económica y tiene demanda } \\
\text { no tenemos una entrada fija de dinero”, E1, E7, E22, E23 “ } \\
\text { un contacto directo con mis vecinos y conocidos un buen } \\
\text { recibimiento me llevo sorpresas por no saber lo que voy a } \\
\text { comer es un ambiente agradable muy cálido”, E5, E10, E23 } \\
\text { “está más céntricoun lugar céntrico”, E3, E7, E9, E12, E13, } \\
\text { E16, E17, E21 “los horarios son muy buenos horarios para } \\
\text { venir a comer acá son flexibles adecuados debe mejorar los } \\
\text { horarios son accesibles” }\end{array}$ \\
\hline Calidad de la dieta & Políticas del servicio \\
\hline $\begin{array}{l}\text { E1, E5, E6, E7, E8, E9, E10, E11, E13, } \\
\text { E14, E15, E17, E18, E19, E20, E22 “está } \\
\text { bienestá bien lo que nos danse ve bien } \\
\text { rico todo", E1, E3, E5, E7, E14, E23 “es } \\
\text { muy variadoson variados los menús y a } \\
\text { veces tengo oportunidad de probar otras } \\
\text { alternativas”, E3, E6, E9, E10, E17, E18, } \\
\text { "hay personas que tienen sus alimen- } \\
\text { taciones especialescon poca grasa”, } \\
\text { E1, E5, E16, "se ha procurado la comida } \\
\text { ponerle menos salni muy salados ni muy } \\
\text { dulces menos azúcar", , E3, E6, E10, E13, } \\
\text { E19 "es poca verdurafruta casi no dan”, } \\
\text { E1, E3, E16, "es un menú muy básico". }\end{array}$ & $\begin{array}{l}\text { E5, E7, E8, E9, E10, E11, E12, E16, E18, E19, E20 “para } \\
\text { la economíaya es un ahorro para la economía } 0 \text { obtener } \\
\text { comida a bajo costo”, E3, E6, E7, E9, E11, E19 “el gobierno } \\
\text { manda todo 0 y de ahí se da la cooperación voluntaria”, E5, } \\
\text { E8, E9, E10, E12, 13, E16, E17, E18, E13, E14 “nadase poco } \\
\text { no puedo yo sabercasi nada”, E3, E5, E6, E9, E10, E12, E14, } \\
\text { E17, E20, E21 “es una gran ayuda 0 hasta señoras venían a } \\
\text { comprar comida” }\end{array}$ \\
\hline
\end{tabular}




\begin{tabular}{|c|c|}
\hline Cultura alimentaria & Intercambio de redes socioculturales \\
\hline $\begin{array}{l}\text { E3, E5, E6, E8, E10, E11, E12, E17, E22 } \\
\text { "hay mucha gente que no se adapta a } \\
\text { todo me adapto, menos a no comer”, } \\
\text { E3, E12, E13, E14 “indagar en la cultura } \\
\text { culinaria } 0 \text { en algunas recetas de familia } \\
\text { cada región tienen sus gustos y cos- } \\
\text { tumbres en cuestión de comida”, E5, } \\
\text { E9, E11, E16, E17, E20, E22 “el sazón y } \\
\text { sabor varían no tan buenos porque esas } \\
\text { comidas casi no las consumíamos”, E6, } \\
\text { E12, E26, E18, E22 “ más variedadfalta } \\
\text { otro tipo de alimentos” }\end{array}$ & $\begin{array}{l}\text { E3, E6, E7, E8, E9, E11, E12, E21, “confianzaconvivencia”, } \\
\text { E5, E8, E13, E16, E17, E22 “alegríafelicidad y convivencia } \\
\text { tranquilidadya no tiene uno soledad disfrutando los alimen- } \\
\text { tos”, E3, E7, E9, E10, E11, E13, E14, E16, E17, E18, E19 “un } \\
\text { poco más de difusiónhace falta más darlo a conocer”, E8, } \\
\text { E9, E14, E16 “música para tomar alimentosla televisión es } \\
\text { la que más llama la atención” }\end{array}$ \\
\hline
\end{tabular}

Fuente: Propia.

Figura 4 - Asociación discursiva referente las categorías de los usuarios del Programa Comedores Comunitarios

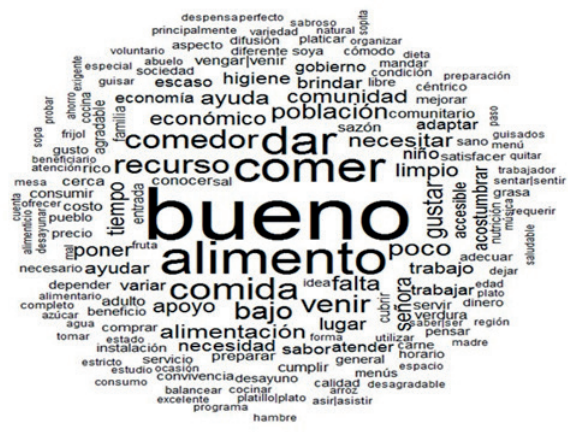

Fuente: Propria.

\subsection{IDENTIDAD}

En esta categoría la comprendemos como la construcción desde el colectivo que define la relación de sí mismos en relación al otro o algo (CASAS; RODRÍGUEZ, 2018), los usuarios comentaron que el CC está asociado a "personas de bajos recursoses un apoyo a la economíael no gastar tantoahorro de tiempobrindan alimento" $\mathrm{E}_{1} \mathrm{E2} 2 \mathrm{E3}, \mathrm{E4}, \mathrm{E} 6, \mathrm{E} 7, \mathrm{E} 8, \mathrm{E} 9, \mathrm{E} 10, \mathrm{E} 11, \mathrm{E} 13, \mathrm{E} 15, \mathrm{E} 16, \mathrm{E} 18, \mathrm{E} 19, \mathrm{E} 20, \mathrm{E} 22, \mathrm{E} 23$, estos discursos están ligados al factor económico y manejo adecuado de los recursos familiares, convirtiéndose en un recursos social, es así que el CC satisface necesidades alimentarias a un bajo costo y con ahorro de tiempo, esto influido por una economía estancada que afecta a colectivos, con esto, se ve expresado que sale mucho más barato ir a este establecimiento para la adquisición de los alimentos a un bajo costo (10 pesos por ración), que comprar con su salario mínimo $\$ 102.68$ (5.29 USD) para la zona centro del país y \$176.72 (9.2USD) en la zona frontera norte (OCCMUNDIAL, 
2018) alimento para la familia, puesto que podemos afirmar que sale más caro cocinar un Kilo de frijol 38 pesos el kilo y 1 litro de gas 9.45 pesos (CASAS; RODRÍGUEZ, 2018) que acudir al CC.

Asimismo, hacen mención que el comedor comunitario es "[] para todo tipo de personas, niños, adultos[]” E4, E5,E6, E15, E16, E17 E19, Е21, Е3, Е5, Е8, E11, Е13, E18, Е22, referido a esto, el Diario Oficial de la Federación (DOF, 2017), especifico desde un inicio una población objetivo, poniendo de manifiesto que se cumple con esta característica: niñas/os de 0 a 11 años de edad, estudiantes adolescentes de 12 a 19 años, mujeres embarazadas y en periodo de lactancia, personas con alguna discapacidad, personas mayores de 65 años y personas en situación de vulnerabilidad, es un programa con accesibilidad percibida en el colectivo (ARAGALL, 2010).

Los usuarios hacen mención que su “[] alimentación es un poco más completapara la nutrición[]" E12, E14, E20, de acuerdo a la Secretaria de Salud (2017), “una buena alimentación significa que tu cuerpo obtiene todos los nutrientes, vitaminas y minerales que necesita para trabajar correctamente", también las leyes de la alimentación dictan que la dieta debe de ser, suficiente, completa, equilibrada, adecuada, variada e higiénica, si esto fuera correcto en el CC se debería de cumplir con estos preceptos, pero en la realidad predominan dietas poco equilibradas y que no necesariamente resultan saludables, no diferenciadas por etapa de vida o condición física, limitadas en términos de variedad, y la preparación de las mismas en condiciones poco adecuadas (HERNÁNDEZ et al., 2015).

Es así que la identidad se construye a partir de economía, nutrición y acceso abierto, esto significa que el CC, es parte del colectivo, ayuda en momentos de pobreza alimentaria y es un brazo largo del Estado, el cual contempla la necesidad alimentaria del colectivo y la palia solo el hambre, sin importarle preceptos nutricios, actividades culturales culinarias y mucho menos, necesidades básicas de participación social.

\subsection{ACCESIBILIDAD}

Entendemos esta categoría como la característica que permite que los entornos, los productos, y los servicios sean utilizados sin problemas por todas y cada una de las personas (ARAGALL, 2010), los usuarios hacen mención que en los CC “[...]es libre porque es comunitariopersonas pobres horarios flexibles accesibles” E3, E6, E7, E8, E9, E12, E13, E16, E17, E18, E19, E21, en estos discursos observamos la perpetuación de mecanismos de trampas de pobreza (NURKSE, 1953; DELAJARA, 2016), los cuales muestran la línea de un programa social de combate a la pobreza como comunitario libre y accesible, construyendo en el imaginario social el rol de Estado benefactor.

Los usuarios hacen mención que existe “[...] un buen recibimiento[...]” y además “[...]es un ambiente agradable muy cálido[...]” E1,E7, E22, E23, los proveedores en los CC son parte de la comunidad, aquí se observa un sitio donde los oprimidos y excluidos se constituyen como parte de un posible cambio sustancial de consecuencia real, donde una política social a través del alimento los convierte en actores políticos de su comunidad.

En esta categoría de accesibilidad se delimitan cinco aspectos fundamentales, libre acceso, pobres, comunitario, cálido y flexibilidad de horarios, con esto podemos observar que los colectivos tienen un programa social que cumple con los criterios de accesibilidad de programa social de acuerdo a European Concept for Accessibility (ECA): trato respetuoso, seguro, saludable, funcional, comprensible, información clara, distribución espacial, estético (ARAGALL, 2010), y además este puede transitar por parte de los proveedores a ser el actor político de cambio en la comunidad. 


\subsection{CALIDAD DE LA DIETA}

Esta categoría se entiende como el conjunto de características basadas en los índices de nutrientes y alimentos combinados de acuerdo a las leyes de la alimentación que satisfacen necesidades alimentarias y nutricionales (CORREIRA et al., 2012; GIL et al., 2015). De acuerdo a lo anterior, la relación discursiva del colectivo gira entorno a “[...] están biense ve bien rico todomuy variado [...]” E1, E3, E5, E6, E7, E8, E9, E10, E11, E13, E14, E15, E17, E18, E19, E20, E22, E23, respeto a esto Hebinck y colaboradores (2018) hace mención que hay aspectos de mala nutrición condicionados por la pobreza, donde la asignación de una dieta de característica barata, con buen sabor y de gratuidad, termina por invisibilisar las características básicas de la dieta saludable, que dote de nutrientes y energía, para la vida diaria, agregan “[...]es un menú muy básicoes poca verdurafruta casi no dan[...]” E1, E3, E16, E10, E13, E19, con lo cual el concepto de hambre es combatido con excelsitud por parte de este programa social, donde las dietas no cubren ni contemplan las calorías, y mucho menos los nutrientes para cada grupo de edad (BALAM et al., 2013).

Los discursos de los usuarios encaminados a "[...]personas que tienen sus alimentaciones especiales

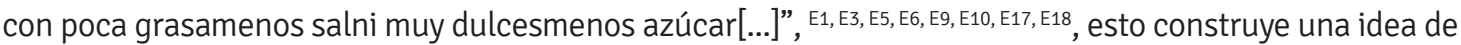
que la mayoría de las personas que acuden al CC, presentan alguna enfermedad crónico no trasmisible que de alguna manera los voluntarios del CC contemplan en el servicio, o esto podria explicar que existe un autocuidado comunitario que se vería gestado en estos CC (OTERO, 2012; ALARCÓN et al., 2017).

Es así que la alimentación se edificó como combate al hambre y autocuidado comunitario, simbolizando que el CC cubre la necesidad básica del hambre al suprimirla o quitarla, pero no hay un enfoque claro de una dieta saludable, y por el otro lado como respuesta social organizada los proveedores del CC cuidan a sus conocidos al procurar alimentos bajos en sal, azúcar y grasas, esta respuesta llamad autocuidado comunitario.

\subsection{POĹTITCAS DEL SERVICIO}

Esta categoría la entenderemos como a las normas o lineamientos del programa CC 2017, los discursos exponen que la "economíaes un ahorrocomida de bajo costo" Е5, Е7, Е8, Е9, Е10, Е11, Е12, Е16, Е18, Е19, Е20 en relación a esto, Medina (2017) menciona que los CC tienen la obligación de proporcionar alimentos a los habitantes más necesitados, pero no se especifica qué tipo de alimentos están contemplados en la dieta diaria o basados en que requerimientos nutricionales de basan para proporcionarlos.

Además los usuarios opinan que “[...] el gobierno manda todouna gran ayuda [...]”Е3, E5, E6, E7, Е8, E9, Е10, E11, E12, E13, E14, E16, E17, E18, E19, E20, E21, esto refleja un bajo nivel de información de los usuarios respecto al funcionamiento del CC, también podemos observar que el deber del Estado respecto a la seguridad alimentaria y la lucha contra el hambre, se prestablece como una política social de gran ayuda para los colectivos, pero esto se puede también analizar como una cuestión de “[...] fines electorales, policiales y judiciales" (LUTZ; TORRES, 2016 p. 171), asociados a un programa social asistencial de carácter nutricional.

En esta categoría de políticas de servicio se demarcan tres elementos; comida de bajo costo, gobierno y proteccionismo, esto significa que el CC es un apoyo del Estado el cual garantiza protección y control, a través de un alimento que combate al hambre pero que en términos nutricionales hay la excepción nutrimental, siendo el CC paliativo del hambre y sub-sanador de olvidos sociales. 


\subsection{CULTURA ALIMENTARIA}

La cultura alimentaria se entiende como los usos (lujo, placer), tradiciones (religión), costumbres (estatus social, interacción social) y elaboraciones simbólicas de los alimentos (MARÍN et al., 2004 apud CALDERÓN et al., 2017).

En esta categoría se encuentran discursos exponiendo que "[]mucha gente que no se adaptaa todo me adapto, menos a no comercada región tienen sus gustos y costumbres en cuestión de comida casi no las consumíamos falta otro tipo de alimentos" E3, E5, E6, E8, E9, E10, E11, E12, E13, E14, E16, E17, E18, E20, E22, LoS discursos hablan de una dieta obligatoria, homogénea y diferente a su cultura, condicionando una dieta no convencional (NGO DE LA CRUZ, 2012), lo que origina que la dieta tradicional como forma de identidad cultural de modifique hacia alimentos poco ordinarios de su dieta, a esto se le confiere un biocontrol alimentario, consumado en los CC.

Aunado a lo anterior, discursos como "[...] indagar en la cultura culinaria en algunas recetas de familia el sazón y sabor varían,esas comidas casi no las consumíamos [...]”, E3, E5, E9, E11, E12, E13, E14, E16, E17, E20, E22 en relación a ello, la cultura alimentaria de una región puede ser elemento clave en la planificación, gestión y efectividad, de los programas sociales diseñados para mejorar la nutrición de una población, esta es determinante en los comportamientos alimentarios y, consecuentemente, en el proceso salud-enfermedad de los individuos y de las comunidades (RICHES 2003 apud GVION, 2006; CALDERÓN et al., 2017; GÓMEZ; VELÁZQUEZ., 2019), aquí observamos que la dieta en el CC transita hacia el olvido de una cultura culinaria donde la reproducción social e identidad se desvanecen como bien cultural.

En esta categoría de cultura alimentaria se proyectó una dieta no convencional, biocontrol alimentario y perdida de cultura culinaria, en relación a esto, se puede entender que el CC adecua menús dietéticos de manera homogénea, no apegada a estructuras culturales de la zona y mucho menos rescata la cultura alimentaria del país.

\subsection{INTERCAMBIO DE REDES SOCIOCULTURALES}

Se entiende a esta categoría como la comunicación, el sentimiento, la confianza, etc. que se trasmiten en un grupo determinado de persona a persona en un espacio social. Respecto a esta categoría los usuarios hacen noción a que el comedor comunitario les da "[] confianza convivencia alegría felicidad tranquilidad ya no tiene uno soledad disfrutando los alimentos []" E3, E5, E6, E7, E8, E9, E11, E12, E13, E14, E16, E17, E18, E19, E21, E22, respecto a esto, el CC condiciono de acuerdo a los discursos ambientes socializadores y patrones asociativos, cabe mencionar que pacientes que cursan con diabetes mellitus u obesidad mejoran sus cifras bioquímicas al comer en compañía familiar (DÍAZ, 2014; RODRÍGUEZ et al., 2014), es así que el CC es un apoyo social contemplado por los usuarios, de esta forma la estructura de programa social brinda un soporte Estatal.

También refieren que “[] se necesita música para tomar los alimentos []” E8, E9, E14, E16, esto concuerda a lo que dice Huergo (2016), que "más allá de comer, se bailaba, tocaba la guitarra, jugaba a las bochas y a las cartas”, el alimento es la anergia para el cuerpo y la música alma de la vida. 
Esta categoría se construyó a partir de apoyo social, el CC brinda un espacio social el cual se ha convertido en un territorio del colectivo donde se intercambian simbolismos propios de la comunidad: reconocimiento, alegría, felicidad, identidad, con las demás personas, y no solamente al acto de alimentarse.

\subsection{ANÁLISIS SOCIO CRÍTICO: BASADOS EN ASOCIACIÓN DISCURSIVA}

La relación discursiva, se esquematizo encontrándose un nucleó y una periferia relacionada al programa CC (FIGURA 4), el núcleo de asociación transcurre entre bueno, alimento, recurso, comida, gustar, limpio, población, comedor, comunidad, limpio, económico, sazón, sabor, beneficio, entre otros, esta fortaleza de significados en torno al CC obliga a conducir que el programa social CC se estableció ante una necesidad muy importante del país "hambre", y que en definitiva el colectivo apropio como buena y satisfactoria, en torno a la periferia de la asociación, convivencia, hambre, programa, ahorro, necesario, preparación, saludable, región, calidad, entre otros, el imaginario colectivo se dibuja con buenas perspectivas y opiniones, cabe mencionar que los colectivos refuerzan la necesidad de los CC como parte fundamental de su desarrollo.

Esto nos lleva al análisis desde la política social, los CC se reconfiguraron como esa parte asistencial de combate a la pobreza alimentaria, porque doto de provisión de servicios comunitarios, en este caso alimentario, con excelente cobertura nacional y de buena calidad percibida por los usuarios siendo esto un desarrollo excelente de una política social, y además construyo un territorio social para la búsqueda de identidad. A esto, las políticas públicas se siguen pensando desde parámetros ajenos a las realidades propias del país, planeándolas de manera desvinculada del contexto de realidades que enmarcan las posibilidades subjetivas y objetivas (GÓMEZ, 2013). Partiendo de que el hambre es el signo absoluto de los vulnerables y puede convertirse en un medio para que los desamparados formen lazos sociales dentro de la comunidad (ZAMUDIO et al., 2018).

Ahora bien, desde el aspecto nutricional los CC sometieron a los colectivos a una dieta no convencional, homogénea a nivel nacional, no se contempló cultura culinaria, nunca se contempló a los productores y productos alimentarios de la zona, las dietas fueron estructuradas en el Centro del país por nutriólogos militares (HERNÁNDEZ et al., 2015), el suministro de los alimentos conto con la ayuda de la iniciativa privada (Neoliberalismo en programa social), con lo cual solo se palio el "hambre".

Los colectivos deben salvar sus espacios de apropiación social, los CC brindaron una herramienta social la cual innovo, al penetrar en lugares de difícil acceso, mejoro relaciones sociales de conflicto al integrar colectividad a partir del alimento, nunca existió dentro de los CC clientelismo político Estatal, puesto que el CC fue abierto a toda la comunidad sin restricción de clero, partido político o condición social, por lo cual, el colectivo debe intervenir en el rescate de un CC integral, donde se dote de alimento con alto aporte nutricional, que sea dotado o suministrado por comerciantes locales o regionales, llevando una cultura culinaria de la comunidad o región, en la búsqueda de CC con desarrollo social, aquí el CC puede brindar participación social y activismo político, al ser un territorio construido alrededor del alimento, como en aquellas épocas donde transitaba la vida alrededor de la mesa de la comida. Ante esto, actualmente la 4T encabezada por el presidente Andrés Manuel López Obrador, en su conferencia de prensa mañanera afirmo que el retito del presupuesto para los CC es 
para una limpia de programas sociales, ya que solo mediatizaban, incurrían en corrupción, utilizando el nombre de gente humilde y gente pobre (SUN, 2019)

\section{CONCLUSIONES}

Desde una perspectiva de análisis socio crítico los CC son una ayuda alimentaria, y durante el tiempo han representado un sustento paliativo directamente enfocado al hambre más no a la nutrición. Existen elementos que se pueden discutir, en este sentido la realidad social de los usuarios frente a la política social del programa CC está construida sobre un núcleo central discursivo dominante a partir de la economía mostrándose en apoyo social y proteccionismo estatal, al brindar una dieta rica, con acceso abierto y libre, cálido, para pobres, desprotegidos, con flexibilidad de horario, esto construye un imaginario de que los CC son del Estado para brindar ayuda alimentaria a las comunidades, con esto la política social cumple su cometido paliar el hambre, pero la otra realidad invita a la reflexión, donde una dieta no saludable, donde los elementos nutricionales y dadores de energía, son suprimidos y olvidados por completo, es así que el CC a través de una necesidad básica realiza biocontrol en las comunidades más paupérrimas del México olvidado. Siendo una política desvinculada y ajena al contexto de las realidades propias del continente.

Respecto a lo anterior, se ha desentendido la garantía del derecho a la alimentación (adecuada, de calidad, completa, inocua, suficiente y variada) principalmente a su población objetivo. Gran parte de esto se debe a la rápida expansión de la cobertura del programa, ya que se tiene el acceso a los alimentos, pero no resuelve ni un problema de fondo en este caso el hambre, descuidando el componente de nutrición principalmente, buscando el beneficio propio del Estado, teniendo como cuartada el contribuir a lograr los Objetivos del Milenio en erradicar la pobreza y el hambre.

Cabe mencionar que actualmente en la 4T encabezada por el gobierno de Andrés Manuel López Obrador están desapareciendo los CC en base a una limpia de programas sociales, pero se invita a que puedan volver a resurgir ya que son una buena iniciativa, replanteando su estrategia de funcionamiento, llevado a cabo por medio de la respuesta social organizada dentro de este programa, con ello logrando un programa de autogestión de seguridad alimentaria, donde se brinde una alimentación digna, cumpliendo con los indicadores de calidad de la dieta, así mismo sea acorde a su cultura.

\section{BIBLIOGRAFÍA}

ALARCÓN, C. et al. Apoyo social y su asociación con el autocuidado de la dieta en personas con diabetes. Liberabit, v. 23, n. 1, p. 110-122, 2017

APARICIO, A. Valores, prácticas y creencias de los católicos mexicanos: un estudio empírico con datos de la Encuesta Mundial de Valores. Rev Iberoameric Teol, v. 11, n. 20, p. 55-78, 2015. 
ARAGALL, F. La accesibilidad en los centros educativos. España. Ediciones cinca. 2010. Disponible en: https://www.cermi.es/sites/default/files/docs/colecciones/LA_ACCESIBILIDAD_EN_LOS_ CENTROS_EDUCATIVOS.pdf. Accedido en: 7 oct. 2020.

BALAM, M. et al. Evaluación de los comedores comunitarios en Tizimín, Yucatán, México: percepciones y propuestas del personal y beneficiarios. Enferm Universit, v. 10, n. 4, p. 125-132, 2013.

BOURDIEU, P. Sobre el Estado. Cursos en el Collège de France (1989-1992). Rev Cien Soc, v. 3, n. 1, p. 147-148, 2014.

CALDERÓN, M. E. et al. Cultura alimentaria: Clave para el diseño de estrategias de mejoramiento nutricional de poblaciones rurales. Agricult Soc Des, v. 14, n. 2, p. 303-320, 2017.

CANSINO, C.; COVARRUBIAS, I. Retoricas y dinámicas del populismo en México: un análisis desde la teoría política. Rev Enfoques Cien Pol Adm Públ, n. 7, p. 37-72, 2007.

CASAS, D.; RODRÍGUEZ, A. Representaciones sociales del sector salud mexicano: la voz de los usuarios. Interf Cient Saude Amb, v. 6, n. 3, 9-28, 2018.

CASTRONUOVO, L. et al. Las madres y la publicidad de alimentos dirigida a niños y niñas: percepciones y experiencias. Salud Colect, v. 12, n. 4, p. 537-550, 2016.

CONEVAL - Consejo Nacional de Evaluación de la Política de Desarrollo Social. Comunicado de prensa, n. 006/09, 2009.

CONEVAL - Consejo Nacional de Evaluación de la Política de Desarrollo Social. Informe de pobreza en México, 2012.

CONEVAL - Consejo Nacional de Evaluación de la Política de Desarrollo Social. Ficha de monitoreo 2016 - 2017. 2017. Disponible en: https://www.coneval.org.mx/Evaluacion/Documents/ EVALUACIONES/FMyE_2016_2017/FMyE_20_U009.pdf. Accedido en: 7 oct. 2020.

CONTRERAS, J. Alimentación y religión. Barcelona: Humanitas humanidades Médicas, 2007.

CORREIA, G. et al. Gestión de calidad del servicio de alimentos y bebidas. La importancia del manipulador de alimentos en la calidad del servicio hotelero de la ciudad de João Pessoa, Brasil. Est Perspec Turismo, v. 21, n. 3, p. 763-777, 2012.

DELAJARA. M. Millones inactivos por exclusión social. Forbes, Ed. 9 de noviembre de 2016.Disponible en: https://www.forbes.com.mx/millones-inactivos-por-exclusion- social/. Accedido en: 7 oct. 2020 
DÍAZ, A. La SEDESOL bajo Enrique Peña Nieto. Revista Nexos. Ed. 11 octubre de 2018. Disponible en: https://economia.nexos.com.mx/?p=1921. Accedido em: 25 set. 2019.

DíAZ, J. A. Reflexiones en tono al concepto de Religión. Est Filosofía, v. 51, p. 27-43, 2015.

DÍAZ, M. P. Factores influyentes en el comportamiento alimentario infantil. Rev Fac Med, v. 62, n. 2, p. 237-245, 2014.

DOF - Diario Oficial de la Federación. Programa nacional México sin hambre 2014-2018. Gobierno Federal. 2014. Disponible en: http://www.dof.gob.mx/nota_detalle_popup.php?codigo=5343098. Accedido en: 29 abr. 2019.

DOF - Diario Oficial de la Federación. Acuerdo por el que se emiten las reglas de operación del programa comedores comunitarios para el ejercicio fiscal 2018. 2017. Disponible en: http://www. dof.gob.mx/nota_detalle.php?codigo=5509780\&fecha=30/12/2017. Accedido en: 29 abr. 2019 .

ENSANUT - Encuesta Nacional de Salud y Nutrición de Medio Camino 2016. Informe final de resultados. México: Instituto Nacional de Salud Pública, 2016.

FERNÁNDEZ, S. Consideraciones de la teoría sociocrítica de la enseñanza. Enseñanza, v. 13, p. 241255, 1995.

GARZA, N. P. et al. Impacto del Programa Comedores Comunitarios SINHAMBRE sobre la desnutrición en Chiapas rural mediante el enfoque de seguridad Alimentaria. Pobl Salud Mesoam, v. 16, n. 1, p. 1-31, 2018.

GIL, A. et al. Indicadores de evaluación de la calidad de la dieta. Rev Esp Nutr Comunit, v. 21, n. 1, p. 127-143, 2015.

GÓMEZ, F. Políticas publicas críticas para y desde América Latina. Pol Cult, v. 40, p. 79-98, 2013.

GÓMEZ, M. E. Panorama del sistema educativo mexicano desde la perspectiva de las políticas públicas. Innov Educ, v. 17, n. 74, p. 143-163, 2017.

GÓMEZ, Y., VELÁZQUEZ, E. B. Salud y cultura alimentaria en México. Rev Dig Universit, v. 20, n. 1, 2019. Disponible en: http://doi.org/10.22201/codeic.16076079e.2019.v20n1.a6. Accedido en: 17 jul. 2020.

GVION, L. Cuisines of poverty as means of empowerment: arab food in Israel. Agricult Human Val, $n$. 23, p. 299-312, 2006. 
HEBINCK, A. et al. Capturing change in European food assistance practices: a transformative social innovation perspective. Local Environm, v. 23, n. 4, p. 398-413, 2018.

HERNÁNDEZ, C. et al. El programa de comedores comunitarios: análisis de su diseño e instrumentación. Programa Universitarios de Estudios del Desarrollo. Documento de Trabajo v. 16, agosto 2015.

HUERGO, J. "Darse un gustito”, cocinar y comer en familia. Villa La Tela, Córdoba, Argentina. Physis Rev Saúde Col, v. 26, n. 4, p. 1125-1159, 2016.

INEGI - Instituto Nacional de Estadística y Geografía. Los jóvenes en el estado de México. México, 2009. Disponible en: https://www.codhem.org.mx/LocalUser/codhem.org/infantil/pdf/ jovenesedomex.pdf. Accedido en: 14 ago. 2019.

JIMÉNEZ, F. et al. Una aproximación microetnográfica de prácticas pedagógicas en escuelas multiculturales. Perf Educ, v. 39, n. 156, p. 72-88, 2017.

JIMÉNEZ, N. De 6 mil 117 pesos al mes, el salario promedio. La Jornada, Ed. 22 de octubre de 2018. Disponible en: file:///E:/Articulos/La\%20Jornada,\%202018.html. Accedido en: 7 oct. 2020.

LERULLO, M. Prácticas de cuidado infantil en organizaciones comunitarias. Los comedores comunitarios en el Área Metropolitana de Buenos Aires, Argentina. Portularia, v. 13, n. 1, p. 59-65, 2013.

LOOPSTRA, R., TARASUK, V. Perspectives on community gardens, community kitchens and the Good Food Box program in a community-based sample of low-income families. Can J Publ Health, v. 104, n. 1, p. 55-59, 2013.

LUTZ, B., TORRES, F. Las fuerzas armadas en los comedores comunitarios del programa sin hambre. El caso de la montaña de Guerrero. Rev Cien Soc Univer Iberoam, v. 11, n. 22, p. 150-177, 2016.

MARTÍNEZ, C. El muestreo en investigación cualitativa. Principios básicos y algunas controversias. Ciên Saúde Col, v. 17, n. 3, p. 613-619, 2012.

MARTÍNEZ, D. Los 5 programas sociales que quedaron relegados del Presupuesto 2019. Expansión, Ed. 27 de enero de 2019. Disponible en: https://politica.expansion.mx/congreso/2019/01/27/ programas-sociales-relegados-presupuesto-2019. Accedido en: 3 ago. 2020.

MARTÍNEZ, T. México registra el crecimiento económico más bajo en 4 años. El economista, Ed. 23 de febrero de 2018. Disponible en: https://www.eleconomista.com.mx/economia/Mexico-registrael-crecimiento-economico-mas-bajo-en-4-anos-20180223-0057.html. Accedido en: 7 oct. 2020. 
MEDINA, A.M. Los comedores comunitarios como alternativa para combatir el hambre en México. Estudio de caso San Felipe Tlalmimilolpan, Toluca, Estado de México 2013-2016. 2017. 139 f. TCC

(Ciencias Políticas y Administración Pública) - Universidad Autónoma del Estado de México, México, 2017.

MILLS, S. et al. Health and social determinants and outcomes of home cooking: A systematic review of observational studies. Appetite, v. 111, p. 116-134, 2017.

MOCTEZUMA, D. et al. La mujer en México: inequidad, pobreza y violencia. Rev Mex Cien Pol Soc, v. 59, n. 220, p. 117-146, 2014.

MORA, Y. Costo de la canasta básica se triplica en 14 años: UdeG. Informador, Ed. 8 de julio 2019. Disponible en: https://www.informador.mx/economia/Costo-de-la-canasta-basica-se-triplica-en-14anos-UdeG-20190708-0089.html. Accedido en: 3 ago. 2020.

NGO DE LA CRUZ, J. Alimentación en otras culturas y dietas no convencionales. Martin. (Presidencia). IX Curso de Actualización Pediatría. Madrid, España: Exlibris Ediciones, 2012.

NOTIMEX - Agencia de Noticias del Estado Mexicano. México cuenta con 123.5 millones de habitantes. El economista, Ed. 10 de julio de 2017. Disponible en: https://www.eleconomista.com.mx/politica/ Mexico-cuenta- con-123.5-millones-de-habitantes-20170710-0116.html. Accedido en: 29 abr. 2020.

NURKSE, R. Problems of capital formation in underdeveloped countries. New York: Oxford University Press. 1957

OCCMUNDIAL. Salario mínimo 2019: ¿para que alcanza con 102 pesos? Fecha de consulta: 21 de marzo 2018. Disponible en: https://www.occ.com.mx/blog/salario-minimo-2019-en-mexico/. Accedido en: 21 mar. 2018.

OSSA, D.A.; MUÑOZ, I.F. Explotación sexual comercial de niños, niñas y adolescentes: significados y prácticas de trabajadores/as y residentes del centro de Medellín. Salud Col, v. 13, n. 1, p. 19-34, 2015.

OTERO, B. Nutrición. Estado de México, México: Red Tercer Milenio, 2012.

RODRÍGUEZ, A. et al. Representación social del apoyo familiar al diabético en usuarios de una unidad de medicina familiar en Chalco, Estado de México. Medwave, v. 14, n. 7, e6011, 2014.

ROSAS, A. La pobreza en México daña más a las mujeres y los indígenas. Expansión, Ed 6 de agosto de 2019. Disponible en: https://www.msn.com/es-mx/dinero/noticias/la-pobreza-enm\%C3\%A9xico-da\%C3\%B1a-m\%C3\%A1s-a-las-mujeres- y-los-ind\%C3\%ADgenas/ar-AAFpZxX. Accedido en: 3 ago. 2020. 
SECRETARIA DE BIENESTAR. Preguntas Frecuentes de los Trámites del Programa de Comedores Comunitarios. 2018. Disponible en: https://www.gob.mx/bienestar/documentos/preguntasfrecuentes-de-los-tramites-del-programa-de-comedores-comunitarios. Accedido en: 14 ago. 2019.

SECRETARIA DE SALUD. El plato del bien comer una guía para una buena alimentación. 2017. Disponible en: https://www.gob.mx/salud/es/articulos/el-plato-del-bien-comer-una-guia-para-unabuena-alimentacion Accedido en: 23 oct. 2019.

SECRETARÍA DE DESARROLLO SOCIAL. Cruzada nacional contra el Hambre. 2013. Disponible en: https://www.gob.mx/cms/uploads/attachment/file/120919/CruzadaNacionalContraElHam bre.pdf. Accedido en: 7 abr. 2020.

SERBIA, J. M. Diseño, muestreo y análisis en la investigación cualitativa. Rev Cien Soc Comunic, v. 3, n. 29, p. 123-146, 2019.

SUN. Retiro de comedores comunitarios, por desvíos de recursos: López obrador. Informador, Ed. 12 de marzo, 2019. Disponible en: https://www.informador.mx/mexico/Retiro-de-comedores-comunitariospor-desvio-de-recursos-Lopez-Obrador-20190312-0056.html. Accedido en: 29 mar. 2020.

TORRES, F.; LUTZ, B. Papel de la industria alimentaria y de la sociedad civil en los comedores comunitarios de SINHAMBRE. Casos de la Montaña y centro de Guerrero. Espiral, v. 23, n. 67, p. 239-277, 2016.

VELÁZQUEZ, E. B. et al. Plagio y usurpación del conocimiento de filosofías científicas. Rev Comunic SEECI, n. extraordinario, p. 134-141, 2014.

ZAMUDIO, A. et al. Las motivaciones para la participación voluntaria en comedores comunitarios del Estado de México. Est Soc, v. 28, n. 52, p. 1-24, 2018. 
1 Doctor en Ciencias de Salud Colectiva; Posdoctor en Antropología Médica y Social; Maestro en Población y Salud; Especialista en Medicina Familiar; Médico Cirujano, adscrito a la Universidad Autónoma del Estado de México CU Amecameca; Integrante de la RED Internacional en Salud Colectiva y Salud Intercultural.

E-mail: capo730211@yahoo.es

2 Maestro en Sociologia de la Salud; Licenciado en Nutricion Clínica, adscrito a la Universidad Autónoma del Estado de México CU Amecameca; Integrante de la RED Internacional en Salud Colectiva y Salud Intercultural.

E-mail: ferrcastro1g@gmail.com

3 Doctora en Ciencias de Salud Colectiva; Posdoctor en Antropología Médica y Social; Maestro en Sociologia de la Salud; Especialista en Medicina Familiar; Médica Cirujano, adscrito al Instituto Mexicano del Seguro Social UMF 73 Amecameca y Universidad Autónoma del Estado de México CU Amecameca; Integrante de la RED Internacional en Salud Colectiva y Salud Intercultural.

E-mail: aledefra2001@yahoo.com.mx

4 Doctora en Ciencias en Salud Colectiva; Maestra en Administración de Sistemas de Salud; Profesora Investigadora de Tiempo Completo en la Licenciatura de Educación para la Salud y Maestría en Sociología de la Salud - UAEM; Integrante del Cuerpo Académico Consolidado (SEP) "Psicología, salud y sociedad"; Integrante de la RED Internacional en Salud Colectiva y Salud Intercultural.

E-mail: gcontreras1l@uaemex.mx

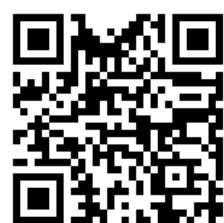

A autenticidade desse artigo pode ser conferida no site https://periodicos. set.edu.br

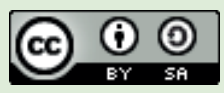

Este artigo é licenciado na modalidade acesso abertosob a Atribuição-Compartilhalgual CC BY-SA
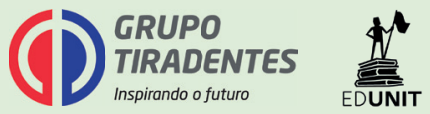